\title{
A Class of Lie 2-Algebras in Higher-Order Courant Algebroids
}

\author{
Yanhui Bi, Fengying Han, Meili Sun \\ College of Mathematics and Information Science, Nanchang Hangkong University, Nanchang, China \\ Email: biyanhui0523@sina.com,tjhanfengying@163.com,sunml8827@163.com
}

Received 9 May 2016; accepted 12 July 2016; published 15 July 2016

\begin{abstract}
In this paper, we study the relation of the algebraic properties of the higher-order Courant bracket and Dorfman bracket on the direct sum bundle $T M \oplus \wedge^{p} T^{*} M$ for an $m$-dimensional smooth manifold $M$, and a Lie 2-algebra which is a "categorified" version of a Lie algebra. We prove that the higher-order Courant algebroids give rise to a semistrict Lie 2 -algebra, and we prove that the higher-order Dorfman algebroids give rise to a hemistrict Lie 2-algebra. Consequently, there is an isomorphism from the higher-order Courant algebroids to the higher-order Dorfman algebroids as Lie 2-algebras homomorphism.
\end{abstract}

Keywords

Higher-Order Courant Algebroids, Higher-Order Dorfman Algebroids, Lie 2-Algebra

\section{Introduction}

The notion of Courant algebroid was introduced in [1] to study the double of Lie bialgebroids. Equivalent definition was given by Roytenberg [2]. In resent years, with the development and exploration of the theory of categorified Lie algebras, or "Lie 2-algebras", Courant algebroids have been far and wide studied from several aspects and have been found many applications in the theory of Manin pairs and moment maps [3] [4]; generalized complex structures [5]; $L_{\infty}$-algebras and symplectic supermanifolds [2]; gerbes [6] as well as BV algebras and topological field theories.

But these articles just introduced the Courant algebroids and Dorfman algebroids. And they did not find the relation between the higher-order Courant algebroids and the higher-order Dorfman algebroids.

The standard Courant algebroid is the direct sum bundle $T M \oplus T^{*} M$. The standard Courant bracket is given by

$$
\llbracket X+\alpha, Y+\beta \rrbracket=[X, Y]+L_{X} \beta-L_{Y} \alpha+\frac{1}{2}\left(d i_{Y} \alpha-d i_{X} \beta\right) .
$$

However, many experts know that on the direct sum bundle $T M \oplus \wedge^{p} T^{*} M$, there is also a similar bracket operation, i.e.

$$
\llbracket X+\alpha, Y+\beta \rrbracket=[X, Y]+L_{X} \beta-L_{Y} \alpha+\frac{1}{2}\left(d i_{Y} \alpha-d i_{X} \beta\right), \quad \forall X, Y \in \mathfrak{X}(M), \alpha, \beta \in \Omega^{p}(M),
$$


which we call the higher-order Courant bracket. We have proved that the Jacobi identity holds up to an exact term.

So in our paper, we introduce the higher-order Courant algebroids and Dorfman algebroids, and find their relation. In Section 2, we review the higher-order Courant bracket and higher-order Courant Dorfman bracket. Also we review the basic definitions about the Dorfman algebroids and Lie 2-algebras. In Section 3, we introduce emphatically the equivalence between the higher-order Courant algebroids and higher-order Dorfman algebroids. In Thm. 3.1, we construct a Lie 2-algebra which is "semistrict", meaning that the bracket is skewsymmetric, but the Jacobi identity holds only up to isomorphism, where the Lie bracket of observables is given instead by the higher-order Courant bracket. In Thm. 3.2, we construct another Lie 2-algebra with the same objects and morphisms, where the Lie bracket of observables is given instead by the higher-order Dorfman bracket. In Thm. 3.3, we show that these two Lie 2-algebras are isomorphic.

\section{Preliminaries}

In this section, we introduce the higher-order Courant and Dorfman bracket on the direct sum bundle $\mathbb{T}^{p} \triangleq T M \oplus \wedge^{p} T^{*} M$ and associated properties. Also we review the definition of Lie 2-algebras.

First, there is a natural $\wedge^{p-1} T^{*} M$-valued nondegenerate symmetric pairing $(\cdot, \cdot)_{+}$on the direct sum bundle $\mathbb{T}^{p}$,

$$
(X+\alpha, Y+\beta)_{+}=\frac{1}{2}\left(i_{X} \beta+i_{Y} \alpha\right), \quad \forall X, Y \in \mathfrak{X}(M), \alpha, \beta \in \Omega^{p}(M) .
$$

The higher-order Courant bracket satisfies some similar properties as the Courant bracket.

Theorem 2.1. [7] For any $e_{1}, e_{2}, e_{3} \in \Gamma\left(\mathbb{T}^{p}\right), f \in C^{\infty}(M), \xi \in \Omega^{p-1}(M)$, we have

1) $\llbracket e_{1}, \llbracket e_{2}, e_{3} \rrbracket \rrbracket+c . p .=d T\left(e_{1}, e_{2}, e_{3}\right)$, where $T: \wedge^{3} \mathbb{T}^{p} \rightarrow \wedge^{p-1} T^{*} M$ is defined by

$$
T\left(e_{1}, e_{2}, e_{3}\right)=-\frac{1}{3}\left(\left(\llbracket e_{1}, e_{2} \rrbracket, e_{3}\right)_{+}+c \cdot p .\right) .
$$

2) $\llbracket e_{1}, f e_{2} \rrbracket=f e_{1}, e_{2}+\rho\left(e_{1}\right)(f) e_{2}-d f \wedge\left(e_{1}, e_{2}\right)_{+}$.

3) $\llbracket \rho e_{1}, e_{2} \rrbracket=\left[\rho\left(e_{1}\right), \rho\left(e_{2}\right)\right]$.

4) $L_{\rho\left(e_{1}\right)}\left(e_{2}, e_{3}\right)=\left(\llbracket e_{1}, e_{2} \rrbracket+d\left(e_{1}, e_{2}\right)_{+}, e_{3}\right)_{+}+\left(e_{2}, \llbracket e_{1}, e_{3} \rrbracket+d\left(e_{1}, e_{3}\right)_{+}\right)_{+}$.

Second, we introduce the following higher-order Dorfman bracket,

$$
\left\{e_{1}, e_{2}\right\}=\llbracket e_{1}, e_{2} \rrbracket+d\left(e_{1}, e_{2}\right)_{+}, \forall e_{1}, e_{2} \in \Gamma\left(\mathbb{T}^{p}\right) .
$$

The higher-order Dorfman bracket also satisfies similar properties as the usual Dorfman bracket.

Theorem 2.2. [7] 1) For any $e_{1}, e_{2} \in \Gamma\left(\mathbb{T}^{p}\right), f \in C^{\infty}(M)$, we have

$$
\left\{e_{1}, f e_{2}\right\}=f\left\{e_{1}, e_{2}\right\}+\rho\left(e_{1}\right)(f) e_{2},\left\{f e_{1}, e_{2}\right\}=f\left\{e_{1}, e_{2}\right\}-\rho\left(e_{2}\right)(f) e_{1}+d f \wedge 2\left(e_{1}, e_{2}\right)_{+} .
$$

2) The Dorfman bracket $\{\cdot, \cdot\}$ is a Leibniz bracket, i.e. for any $e_{1}, e_{2}, e_{3} \in \Gamma\left(\mathbb{T}^{p}\right)$,

$$
\left\{e_{1},\left\{e_{2}, e_{3}\right\}\right\}=\left\{\left\{e_{1}, e_{2}\right\}, e_{3}\right\}+\left(e_{2},\left\{e_{1}, e_{3}\right\}\right)_{+} .
$$

Consequently, $\left(\mathbb{T}^{p},\{\cdot, \cdot\}, \rho\right)$ is a Dorfman algebroid.

3) The pairing (2) and the higher-order Dorfman bracket is compatible in the following sense,

$$
L_{\rho\left(e_{1}\right)}\left(e_{2}, e_{3}\right)_{+}=\left(\left\{e_{1}, e_{2}\right\}, e_{3}\right)_{+}+\left(e_{2},\left\{e_{1}, e_{3}\right\}\right)_{+} .
$$

Third, we review some definitions about the Lie 2-algebra.

Definition 2.3. [8] A Lie 2-algebra is a 2-term chain complex of vector spaces $L=\left(L_{0} \stackrel{d}{\longleftarrow} L_{1}\right)$ equipped with the following structure:

1) A chain map $[\cdot, \cdot]: L \otimes L \rightarrow L$ which called the bracket;

2) A chain homotopy $S:[\cdot, \cdot] \Rightarrow-[\cdot, \cdot] \circ \sigma$ which called the alternator;

3) An antisymmetric chain homotopy $J:[\cdot,[, \cdot]] \Rightarrow[[, \cdot],, \cdot]+[\cdot,[, \cdot],] \circ(\sigma \otimes 1)$ which called the Jacobiator.

In addition, the following diagrams are required to commute: 


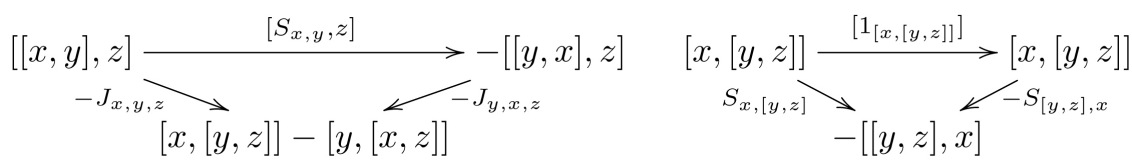

$$
\begin{aligned}
& \left.[x,[y, z]] \longrightarrow-\left[x, S_{y, z}\right] \longrightarrow[z, y]\right] \\
& J_{x, y, z} \quad \downarrow-J_{x, y, z} \\
& {[[x, y], z]+[y,[x, z]] \underset{S_{[x, y], z}+S_{y,[x, z]}}{\longrightarrow}-[[x, z], y]-[z,[x, y]]}
\end{aligned}
$$

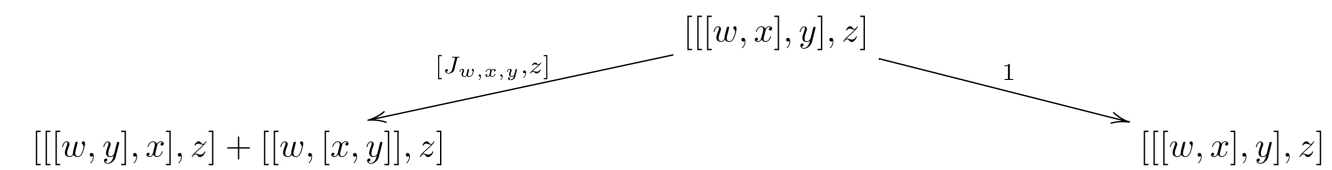

$$
\begin{aligned}
& J_{[w, y], x, z}+J_{w,[x, y], z} \downarrow \\
& {[[[w, y], z], x]+[[w, y],[x, z]]} \\
& +[w,[[x, y], z]]+[[w, z],[x, y]] \\
& {\left[J_{w, y, z}, x\right]+1 \downarrow} \\
& {[[[w, z], y], x]+[[w,[y, z]], x]} \\
& +[[w, y],[x, z]]+[w,[[x, y], z]]+[[w, z],[x, y]] \\
& {\left[w, J_{x, y, z}\right]+1} \\
& {[[[w, z], y], x]+[[w, z],[x, y]]+[[w, y],[x, z]]} \\
& +[w,[[x, z], y]]+[[w,[y, z]], x]+[w,[x,[y, z]]]
\end{aligned}
$$

Definition 2.4. [8] A Lie 2-algebra for which the Jacobiator is the identity chain homotopy is called hemistrict. One for which the alternator is the identity chain homotopy is called semistrict.

Definition 2.5. [8] Given Lie 2-algebras $L$ and $L^{\prime}$ with bracket, alternator and Jacobiator $[\cdot, \cdot], \quad S, J$ and $[\cdot, \cdot]^{\prime}, S^{\prime}, L^{\prime}$ respectively, a homomorphism from $L$ to $L^{\prime}$ consists of:

1) A Chain map $\phi: L \rightarrow L^{\prime}$, and

2) A chain homotopy $\Phi:[\cdot, \cdot]^{\prime} \circ(\phi \otimes \phi) \Rightarrow \phi \circ[\cdot, \cdot]$

such that the following diagrams commute:

$$
\begin{aligned}
& \left.[\phi(x), \phi(y)]^{\prime} \stackrel{\Phi_{x, y}}{\longrightarrow} \phi([x, y]) \quad\left[\phi(x),[\phi(y), \phi(z)]^{\prime}\right]^{J^{\prime}} \stackrel{\phi_{(x), \phi(y), \phi(z)}}{\longrightarrow}[\phi(x), \phi(y)]^{\prime}, \phi(z)\right]^{\prime}+\left[\phi(y),[\phi(x), \phi(z)]^{\prime}\right]^{\prime} \\
& S_{\phi(x), \phi(y)}^{\prime} \downarrow \quad \downarrow \phi\left(S_{x, y}\right) \quad\left[\phi(x), \Phi_{y, z}\right]^{\prime} \downarrow \quad \downarrow\left[\Phi_{x, y}, \phi(z)\right]^{\prime}+\left[\phi(y), \Phi_{x, z}\right]^{\prime} \\
& -[\phi(y), \phi(x)]_{-\Phi_{y, x}^{\prime}}^{\longrightarrow}-\phi([y, x]) \quad[\phi(x), \phi([y, z])]^{\prime} \quad[\phi([y, x]), \phi(z)]^{\prime}+[\phi(y), \phi([x, z])]^{\prime} \\
& \Phi_{x,[y, z]} \downarrow \quad \downarrow \Phi_{[x, y], z}+\Phi_{y,[x, z]} \\
& \phi(x,[y, z]) \longrightarrow \phi([[x, y], z]+[y,[x, z]])
\end{aligned}
$$

\section{The Equivalence between p-Order Courant Algebroid and p-Order Dorfman Algebroid}

In this section, we construct a "semistrict" Lie 2-algebra and a "hemistric" Lie 2-algebra and give the relation between p-order Courant algebroids and p-order Dorfman algebroids. We all know that $\left(\mathbb{T}^{p}, \llbracket \cdot, \cdot \rrbracket, \rho\right)$ is a porder Courant algebroid, and $\left(\mathbb{T}^{p},\{\cdot, \cdot\}, \rho\right)$ is a p-order Dorfman algebroid. We shall construct two Lie 2algebras associated to $\mathbb{T}^{p}$ : one hemistrict and one semistrict. Then we shall prove these are isomorphic. Both these Lie 2-algebras have the same underlying 2-term complex, namely:

$$
L=\mathbb{T}^{p} \stackrel{d}{\longleftarrow} \Omega^{p-1}(M) \stackrel{0}{\longleftarrow} 0 \stackrel{0}{\longleftarrow} 0 \stackrel{0}{\longleftarrow} \cdots
$$

where $\mathrm{d}$ is the usual exterior derivative of functions. To see that this chain complex is well-defined.

We make $L$ into a semistrict Lie 2-algebra. For this, we use a chain map called the semi-bracket: 
$\llbracket \cdot, \cdot \rrbracket: L \otimes L \rightarrow L$. In degree 0, the semi-bracket is given as in Equation (1)

$$
\llbracket X+\alpha, Y+\beta \rrbracket=[X, Y]+L_{X} \beta-L_{Y} \alpha+\frac{1}{2}\left(d i_{Y} \alpha-d i_{X} \beta\right), \quad \forall X, Y \in \mathfrak{X}(M), \alpha, \beta \in \Omega^{p}(M),
$$

In degrees 1 and 2, we set it equal to zero: $\llbracket e_{1}, \xi \rrbracket=\frac{1}{2} i_{X} d \xi=-\llbracket \xi, e_{1} \rrbracket, \llbracket \xi, \eta \rrbracket=0$.

Theorem 3.1. ( $\left.\mathbb{T}^{p}, \llbracket \llbracket \cdot \cdot \rrbracket, \rho\right)$ is a p-order Courant algebroid, there is a semistrict Lie 2-algebra $L\left(\mathbb{T}^{p}, \llbracket \cdot, \cdot \rrbracket, \rho\right)$ where

1) The space of 0 -chains is $\Gamma\left(\mathbb{T}^{p}\right)$;

2) The space of 1-chains is $\Omega^{p-1}(M)$;

3) The differential is the exterior derivative $d: \Omega^{p-1}(M) \rightarrow \Gamma\left(\mathbb{T}^{p}\right)$;

4) The bracket is $\llbracket \cdot \cdot \cdot \rrbracket$;

5) The alternator is the bilinear map $S: \Gamma\left(\mathbb{T}^{p}\right) \times \Gamma\left(\mathbb{T}^{p}\right) \rightarrow \Omega^{p-1}(M)$ defined by $S_{e_{1}, e_{2}}=0$;

6) The Jacobiator is the identity, hence given by the trilinear map $J: \Gamma\left(\mathbb{T}^{p}\right) \times \Gamma\left(\mathbb{T}^{p}\right) \times \Gamma\left(\mathbb{T}^{p}\right) \rightarrow \Omega^{p-1}(M)$ with $J_{e_{1}, e_{2}, e_{3}}=-T\left(e_{1}, e_{2}, e_{3}\right)=\frac{1}{3}\left(\left\langle e_{1}, e_{2}, e_{3}\right\rangle+c . p\right.$. $)$.

Proof. We note from Equation (1) that the semi-bracket is antisymmetric. Since both $S$ and the degree 1 chain map are zero, the alternator defined above is a chain homotopy with the right source and target. So again, we just need to check that the Lie 2-algebra axioms hold. The following identities can be checked by simple calculation, and the commutativity of the last diagram follows:

$$
\begin{aligned}
& \left(\left[e_{4}, J_{e_{1}, e_{2}, e_{3}}\right]+1\right) \circ\left(\left[J_{e_{4}, e_{2}, e_{3}}, e_{1}\right]+1\right) \circ\left(J_{\left[e_{4}, e_{2}\right], e_{1}, e_{3}}+J_{e_{4},\left[e_{1}, e_{2}\right], e_{3}}\right) \circ\left[J_{e_{4}, e_{1}, e_{2}}, e_{3}\right] \\
& =\left(J_{e_{4},\left[e_{1}, e_{3}\right], e_{2}}+J_{\left[e_{4}, e_{3}\right], e_{1}, e_{2}}+J_{\left.e_{4}, e_{1}, e_{2}, e_{3}\right]}\right) \circ\left(\left[J_{e_{4}, e_{1}, e_{3}}, e_{2}\right]+1\right) \circ J_{\left[e_{4}, e_{1}\right], e_{2}, e_{3}} .
\end{aligned}
$$

$\forall e_{1}, e_{2}, e_{3}, e_{4} \in \Gamma\left(\mathbb{T}^{p}\right)$, we have

$$
\begin{aligned}
& \llbracket d T\left(e_{4}, e_{1}, e_{2}\right), e_{3} \rrbracket+d T\left(\llbracket e_{4}, e_{2} \rrbracket, e_{1}, e_{3}\right)+d T\left(e_{4}, \llbracket e_{1}, e_{2} \rrbracket, e_{3}\right) \\
& +\llbracket e_{4}, d T\left(e_{1}, e_{2}, e_{3}\right) \rrbracket+\llbracket d T\left(e_{4}, e_{2}, e_{3}\right), e_{1} \rrbracket \\
& =d T\left(\llbracket e_{4}, e_{1} \rrbracket, e_{2}, e_{3}\right)+\llbracket d T\left(e_{4}, e_{1}, e_{3}\right), e_{2} \rrbracket+d T\left(e_{4}, e_{1}, \llbracket e_{2}, e_{3} \rrbracket\right) \\
& +d T\left(\llbracket e_{4}, e_{3} \rrbracket, e_{1}, e_{2}\right)+d T\left(e_{4}, \llbracket e_{1}, e_{3} \rrbracket, e_{2}\right) .
\end{aligned}
$$

Since the Jacobiator is antisymmetric and the alternator is the identity, the first and second diagrams commute as well. The third diagram commutes because all the edges are identity morphisms.

Next, the hemistrict Lie 2-algebra comes with a bracket called the hemi-bracket: $\{\cdot, \cdot\}: L \times L \rightarrow L$. In degree 0 , the hemi-bracket is given as Dorfman bracket:

$$
\{X+\alpha, Y+\beta\}=[X, Y]+L_{X} \beta-i_{Y} d \alpha
$$

In degree 1 , it is given by: $\{X+\alpha, \xi\}=i_{X} d \xi,\{\eta, Y+\beta\}=0$. In degree 2 , we necessarily have $\{\xi, \eta\}=0$, where $X+\alpha, Y+\beta \in \Gamma\left(\mathbb{T}^{p}\right)$, while $\xi, \eta \in \Omega^{p-1}(M)$.

To see that the hemi-bracket is in fact a chain map, it suffices to check it on hemi-brackets of degree 1 :

$$
d\{X+\alpha, \xi\}=d i_{X} d \xi=L_{X} d \xi=\{X+\alpha, d \xi\}, d\{\xi, X+\alpha\}=0=\{d \xi, X+\alpha\} .
$$

Theorem 3.2. ( $\left.\mathbb{T}^{p},\{\cdot, \cdot\}, \rho\right)$ is a p-order Dorfman algebroid, there is a hemistrict Lie 2-algebra $L\left(\mathbb{T}^{p},\{\cdot, \cdot\}, \rho\right)$ where

1) The space of 0 -chains is $\Gamma\left(\mathbb{T}^{p}\right)$;

2) The space of 1-chains is $\Omega^{p-1}(M)$;

3) The differential is the exterior derivative $d: \Omega^{p-1}(M) \rightarrow \Gamma\left(\mathbb{T}^{p}\right)$;

4) The bracket is $\{\cdot, \cdot\}$;

5) The alternator is the bilinear map $S: \Gamma\left(\mathbb{T}^{p}\right) \times \Gamma\left(\mathbb{T}^{p}\right) \rightarrow \Omega^{p-1}(M)$ defined by $S_{e_{1}, e_{2}}=-2\left(e_{1}, e_{2}\right)_{+}$;

6) The Jacobiator is the identity, hence given by the trilinear map $J: \Gamma\left(\mathbb{T}^{p}\right) \times \Gamma\left(\mathbb{T}^{p}\right) \times \Gamma\left(\mathbb{T}^{p}\right) \rightarrow \Omega^{p-1}(M)$ with $J_{e_{1}, e_{2}, e_{3}}=0$.

Proof That $S$ is a chain homotopy with the right source and target follows from thm. (2.2) and the fact that:

$$
\left\{e_{1}, e_{2}\right\}=-\left\{e_{2}, e_{1}\right\}-d S_{e_{1}, e_{2}}, \quad\left\{e_{1}, \xi\right\}=-\left\{\xi, e_{1}\right\}-S_{e_{1}, d \xi} .
$$


Thm. (2.2) also says that the Jacobi identity holds. The following equations then imply that $J$ is also a chain homotopy with the right source and target:

$$
\begin{gathered}
\left\{e_{1},\left\{e_{2}, \xi\right\}\right\}=\left\{\left\{e_{1}, e_{2}\right\}, \xi\right\}+\left\{e_{2},\left\{e_{1}, \xi\right\}\right\}, \\
\left\{e_{1},\left\{\xi, e_{2}\right\}\right\}=\left\{\left\{e_{1}, \xi\right\}, e_{2}\right\}=\left\{\xi,\left\{e_{1}, e_{2}\right\}\right\}=0, \\
\left\{\xi,\left\{e_{1}, e_{2}\right\}\right\}=\left\{\left\{\xi, e_{1}\right\}, e_{2}\right\}=\left\{e_{1},\left\{\xi, e_{2}\right\}\right\}=0 .
\end{gathered}
$$

So, we just need to check that the Lie 2-algebra axioms hold. The first and the last two diagrams commute since each edge is the identity. The commutativity of the second diagram from thm. (2.2) (3) is shown as follows:

$$
S_{\left\{e_{1}, e_{2}\right\}, e_{3}}+S_{e_{2},\left\{e_{1}, e_{3}\right\}}=2\left(\left\{e_{1}, e_{2}\right\}, e_{3}\right)_{+}+\left(e_{2},\left\{e_{1}, e_{3}\right\}\right)_{+}=2 L_{X}\left(e_{2}, e_{3}\right)_{+}=\left\{e_{1}, S_{e_{2}, e_{3}}\right\}
$$

The third diagram says that $S_{e_{1},\left\{e_{2}, e_{3}\right\}}-S_{\left\{e_{2}, e_{3}\right\}, e_{1}}=0$ and this follows from the fact that the alternator is symmetric: $S_{e_{1}, e_{2}}=S_{e_{2}}$

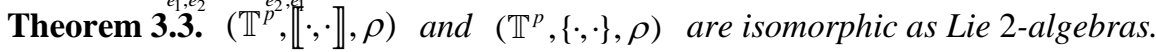

Proof. We show that the identity chain maps with appropriate chain homotopies define Lie 2-algebra homomorphisms and that their composites are the respective identity homomorphisms. There is a homomorphism $\varphi:\left(\mathbb{T}^{p}, \llbracket \cdot, \cdot \mathbb{\rrbracket}, \rho\right) \rightarrow\left(\mathbb{T}^{p},\{\cdot, \cdot\}, \rho\right)$ with the identity chain map and the chain homotopy given by $\Phi_{e_{1}, e_{2}}=\left(e_{1}, e_{2}\right)_{+}$. This is a chain homotopy follows from the bracket relation $\left\{e_{1}, e_{2}\right\}=\llbracket e_{1}, e_{2} \rrbracket+d \Phi_{e_{1}, e_{2}}$, noted in Equation (3) together with the equations

$$
\llbracket e_{1}, \xi \rrbracket+d \Phi_{e_{1}, \xi}=\left\{e_{1}, \xi\right\}, \llbracket \xi, e_{1} \rrbracket+d \Phi_{\xi, e_{1}}=\left\{\xi, e_{1}\right\}
$$

We check that the two diagrams in the definition of a Lie 2-algebra homomorphism commute. Noting that the chain map $\phi$ is the identity, the commutativity of the first diagram is easily checked by recalling that $S_{e_{1}, e_{2}}=2\left(e_{1}, e_{2}\right)_{+}$and $S_{e_{1}, e_{2}}^{\prime}$ are the identities. Noting that any edge given by the bracket for $\left(\mathbb{T}^{p},\{\cdot, \cdot\}, \rho\right)$ in degree 1 is the identity and that $J_{e_{1}, e_{2}, e_{3}}$ is the identity, we easily check the commutativity of the first diagram:

$$
\phi\left(S_{e_{1}, e_{2}}\right) \circ \Phi_{\phi e_{1}, \phi e_{2}}\left(\phi e_{1}, \phi e_{2}\right)=-\llbracket e_{2}, e_{1} \rrbracket+d\left(e_{1}, e_{2}\right)_{+}=-\Phi_{e_{1}, e_{2}} \circ S_{\phi e_{1}, \phi e_{2}}^{\prime}\left(\left\{\phi e_{1}, \phi e_{2}\right\}\right) \text {. }
$$

To check the commutativity of the second diagram we only need to perform the following calculation:

$$
\begin{aligned}
& \left\{e_{1},\left\{e_{2}, e_{3}\right\}\right\} \stackrel{J_{e_{1}, e_{2}, e_{3}}^{\prime}=i d}{\longrightarrow}\left\{\left\{e_{1}, e_{2}\right\}, e_{3}\right\}+\left\{e_{2},\left\{e_{1}, e_{3}\right\}\right\} \\
& \left\{e_{1}, \Phi_{e_{2}, e_{3}}\right\} \downarrow \quad \downarrow\left\{\Phi_{e_{1}, e_{2}}, e_{3}\right\}+\left\{e_{2}, \Phi_{e_{1}, e_{3}}\right\} \\
& \left\{e_{1}, \llbracket e_{2}, e_{3} \rrbracket\right\} \quad\left\{\llbracket e_{2}, e_{1} \rrbracket, e_{3}\right\}+\left\{e_{2}, \llbracket e_{1}, e_{3} \rrbracket\right\} \\
& \Phi_{e_{1}, \llbracket e_{2}, e_{3} \rrbracket} \downarrow \\
& \llbracket e_{1}, \llbracket e_{2}, e_{3} \rrbracket \rrbracket \longrightarrow J_{e_{1}, e_{2}, e_{3}} \longrightarrow \llbracket \llbracket e_{1}, e_{2} \rrbracket, e_{3} \rrbracket+\llbracket e_{2}, \llbracket e_{1}, e_{3} \rrbracket \rrbracket
\end{aligned}
$$

The second diagram meet commutativity if and only if the following equation established:

$$
\begin{aligned}
& \left(\Phi_{\llbracket e_{1}, e_{2} \rrbracket e_{3}}+\Phi_{e_{2}, \llbracket e_{1}, e_{3} \rrbracket}\right) \circ\left(\left\{\Phi_{e_{1}, e_{2}}, e_{3}\right\}+\left\{e_{2}, \Phi_{e_{1}, e_{3}}\right\}\right) \circ J_{e_{1}, e_{2}, e_{3}}^{\prime}\left(\left\{e_{1},\left\{e_{2}, e_{3}\right\}\right\}\right) \\
& =J_{e_{1}, e_{2}, e_{3}} \circ \Phi_{e_{1}, \llbracket e_{2}, e_{3} \rrbracket} \circ\left\{e_{1}, \Phi_{e_{2}, e_{3}}\right\}\left(\left\{e_{1},\left\{e_{2}, e_{3}\right\}\right\}\right)
\end{aligned}
$$

Clockwise from the upper left to the lower right corner:

$$
\begin{aligned}
& \left(\Phi_{\llbracket e_{1}, e_{2} \rrbracket, e_{3}}+\Phi_{e_{2}, \llbracket e_{1}, e_{3} \rrbracket}\right) \circ\left(\left\{\Phi_{e_{1}, e_{2}}, e_{3}\right\}+\left\{e_{2}, \Phi_{e_{1}, e_{3}}\right\}\right) \circ J_{e_{1}, e_{2}, e_{3}}^{\prime}\left(\left\{e_{1},\left\{e_{2}, e_{3}\right\}\right\}\right) \\
& =\left(\Phi_{\llbracket e_{1}, e_{2} \rrbracket, e_{3}}+\Phi_{e_{2}, \llbracket e_{1}, e_{3} \rrbracket}\right) \circ\left(\left\{\Phi_{e_{1}, e_{2}}, e_{3}\right\}+\left\{e_{2}, \Phi_{e_{1}, e_{3}}\right\}\right)\left(\left\{\left\{e_{1}, e_{2}\right\}, e_{3}\right\}+\left\{e_{2},\left\{e_{1}, e_{3}\right\}\right\}\right) \\
& =\left(\Phi_{\llbracket e_{1}, e_{2} \rrbracket, e_{3}}+\Phi_{e_{2}, \llbracket e_{1}, e_{3} \rrbracket}\right)\left(\left\{\llbracket e_{1}, e_{2} \rrbracket, e_{3}\right\}+\left\{e_{2}, \llbracket e_{1}, e_{3} \rrbracket\right\}\right)+\left\{d\left(e_{1}, e_{2}\right)_{+}, e_{3}\right\}+\left\{e_{2}, d\left(e_{1}, e_{3}\right)_{+}\right\} \\
& =\llbracket \llbracket e_{1}, e_{2} \rrbracket, e_{3} \rrbracket+\llbracket \llbracket e_{2}, e_{1} \rrbracket, e_{3} \rrbracket+d\left(\llbracket e_{1}, e_{2} \rrbracket, e_{3}\right)_{+}+d\left(e_{2}, \llbracket e_{1}, e_{3} \rrbracket\right)_{+}+\left\{d\left(e_{1}, e_{2}\right)_{+}, e_{3}\right\}+\left\{e_{2}, d\left(e_{1}, e_{2}\right)_{+}\right\} .
\end{aligned}
$$

Counterclockwise from the upper left to the lower right corner:

$$
\begin{aligned}
& J_{e_{1}, e_{2}, e_{3}} \circ \Phi_{e_{1}, \llbracket e_{2}, e_{3} \rrbracket} \circ\left\{e_{1}, \Phi_{e_{2}, e_{3}}\right\}\left(\left\{e_{1},\left\{e_{2}, e_{3}\right\}\right\}\right) \\
& =J_{e_{1}, e_{2}, e_{3}} \circ \Phi_{e_{1}, \llbracket e_{2}, e_{3} \rrbracket}\left(\left\{e_{1}, \llbracket e_{2}, e_{3} \rrbracket\right\}\right)+\left\{e_{1}, d\left(e_{2}, e_{3}\right)_{+}\right\} \\
& =J_{e_{1}, e_{2}, e_{3}}\left(\llbracket e_{1}, \llbracket e_{2}, e_{3} \rrbracket \rrbracket\right)+d\left(e_{1}, \llbracket e_{2}, e_{3} \rrbracket\right)_{+}+\left\{e_{1}, d\left(e_{2}, e_{3}\right)_{+}\right\} \\
& =\llbracket e_{1}, \llbracket e_{2}, e_{3} \rrbracket \rrbracket+\llbracket e_{2}, \llbracket e_{1}, e_{3} \rrbracket \rrbracket+d T\left(e_{1}, e_{2}, e_{3}\right)+d\left(e_{1}, \llbracket e_{2}, e_{3} \rrbracket\right)_{+}+\left\{e_{1}, d\left(e_{2}, e_{3}\right)_{+}\right\}
\end{aligned}
$$


Because of the commutativity of the second diagram we must have the the following equation:

$$
\begin{aligned}
d T\left(e_{1}, e_{2}, e_{3}\right)+ & d\left(e_{1}, \llbracket e_{2}, e_{3} \rrbracket\right)_{+}+\left\{e_{1}, d\left(e_{2}, e_{3}\right)_{+}\right\} \\
=d\left(\llbracket e_{1}, e_{2} \rrbracket, e_{3}\right)_{+} & +d\left(e_{2}, \llbracket e_{1}, e_{3} \rrbracket\right)_{+}+\left\{d\left(e_{1}, e_{2}\right)_{+}, e_{3}\right\}+\left\{e_{2}, d\left(e_{1}, e_{3}\right)_{+}\right\} \\
d T\left(e_{1}, e_{2}, e_{3}\right)= & d\left(\llbracket e_{1}, e_{2} \rrbracket, e_{3}\right)_{+}+d\left(e_{2}, \llbracket e_{1}, e_{3} \rrbracket\right)_{+}+\left\{d\left(e_{1}, e_{2}\right)_{+}, e_{3}\right\} \\
& +\left\{e_{2}, d\left(e_{1}, e_{3}\right)_{+}\right\}-d\left(e_{1}, \llbracket e_{2}, e_{3} \rrbracket\right)_{+}-\left\{e_{1}, d\left(e_{2}, e_{3}\right)_{+}\right\} \\
= & d\left(\llbracket e_{1}, e_{2} \rrbracket, e_{3}\right)_{+}+d\left(e_{2}, \llbracket e_{1}, e_{3} \rrbracket\right)_{+}-d\left(e_{1}, \llbracket e_{2}, e_{3} \rrbracket\right)_{+} \\
& +d L_{Y}\left(e_{1}, e_{3}\right)_{+}-d L_{X}\left(e_{2}, e_{3}\right)_{+}
\end{aligned}
$$

because of $T\left(e_{1}, e_{2}, e_{3}\right)=-\frac{1}{3}\left(\left(\llbracket e_{1}, e_{2} \rrbracket, e_{3}\right)_{+}+c . p.\right)$, we get the following calculations:

$$
2 d T\left(e_{1}, e_{2}, e_{3}\right)=d\left(e_{1},\left\{e_{2}, e_{3}\right\}\right)_{+}-d\left(e_{2},\left\{\left(e_{1}, e_{3}\right)\right\}\right)_{+}
$$

if we choose $e_{1}=X+\alpha, e_{2}=Y+\beta, e_{3}=Z+\gamma \in \Gamma\left(\mathbb{T}^{p}\right)$,

$$
\begin{aligned}
d\left(e_{1},\left\{e_{2}, e_{3}\right\}\right)_{+}-d\left(e_{2},\left\{\left(e_{1}, e_{3}\right)\right\}\right)_{+} & =\frac{1}{2}\left(-d i_{Y} d i_{Z} \alpha-d i_{Z} d i_{Y} \alpha+2 d i_{Y} i_{Z} d \alpha+c . p .\right) \\
& =2\left(-\frac{1}{4} d i_{Y} d i_{Z} \alpha-d i_{Z} d i_{Y} \alpha+\frac{1}{2} d i_{Y} i_{Z} d \alpha+c . p .\right) \\
& =2 d T\left(e_{1}, e_{2}, e_{3}\right)
\end{aligned}
$$

\section{Acknowledgements}

We give warmest thanks to Zhangju Liu and Yunhe Sheng for useful comments and discussion. We also thanks the referees for very helpful comments.

\section{Funding}

Research was partially supported by NSF of China (11126338, 11461047, 11201218).

\section{References}

[1] Liu, Z.J., Weinstein, A. and Xu, P. (1997) Manin Triples for Lie Bialgebroids. Journal of Differential Geometry, 45, 547-574.

[2] Roytenberg, D. Courant Algebroids, Derived Brackets and Even Symplectic Supermanifolds. PhD Thesis, UC Berkeley. arXiv:math.DG/9910078

[3] Alekseev, A. and Kosmann-Schwarzbach, Y. (2000) Manin Pairs and Moment Maps. Journal of Differential Geometry, 56, 133-165.

[4] Li-Bland, D. and Meinrenken, E. (2009) Courant Algebroids and Poisson Geometry. International Mathematics Research Notices, 11, 2106-2145.

[5] Gualtieri, M. Generalized Complex Geometry. arxiv:math.DG/0401221.

[6] Ševera, P. and Weinstein, A. (2001) Poisson Geometry with a 3-Form Background. Progress of Theoretical Physics Supplements, 144, 145-154. Noncommutative Geometry and String Theory (Yokohama, 2001).

[7] Bi, Y.H. and Sheng, Y.H. (1990) On Higher Analogues of Courant Algebroids. Science China Mathematics A, Preprint.

[8] Roytenberg, D. On weak Lie 2-Algebras. arXiv:0712.3461 